Quim. Nova, Vol. 35, No. 3, 454-459, 2012

\title{
USO DO BIVALVE LÍMNICO Anodontites tenebricosus (LEA, 1834) NO BIOMONITORAMENTO DE METAIS DO RIO RIBEIRA DE IGUAPE
}

\author{
Valéria Guimarães Silvestre Rodrigues* \\ Departamento de Geotecnia, Escola de Engenharia de São Carlos, Universidade de São Paulo, Av. Trabalhador São-carlense, 400 , \\ 13566-590 São Carlos - SP, Brasil \\ Aline Fujikawa e Denis Moledo de Souza Abessa \\ Universidade Estadual Paulista "Júlio de Mesquita Filho", Campus Experimental do Litoral Paulista, Pça. Infante D. Henrique, \\ s/no, $11330-900$ São Vicente - SP, Brasil \\ Marcos Antonio Hortellani e Jorge Eduardo Souza Sarkis \\ Instituto de Pesquisas Energéticas e Nucleares, Av. Lineu Prestes, 2242, 05508-000 São Paulo - SP, Brasil \\ Joel Barbujiani Sígolo \\ Departamento de Geologia Sedimentar e Ambiental, Instituto de Geociências, Universidade de São Paulo, Rua do Lago, 562 , \\ 04280-000 São Paulo - SP, Brasil
}

Recebido em 2/3/11; aceito em 8/9/11; publicado na web em 8/11/11

\begin{abstract}
USING THE FRESHWATER BIVALVE Anodontites tenebricosus (LEA, 1834) AS A BIOMONITOR OF METALS IN THE RIBEIRA DE IGUAPE RIVER. This study investigated the contamination of the Ribeira de Iguape River - RIR by $\mathrm{Cd}, \mathrm{Zn}, \mathrm{Cr}$ and $\mathrm{Pb}$, using the bivalve Anodontites tenebricosus as a biomonitor. Metal concentrations in tissue samples were measured by HR-ICPMS. Bivalve tissues exhibited mean levels of $1.00 \mu \mathrm{g} / \mathrm{g} \mathrm{Cd} ; 152.89 \mu \mathrm{g} / \mathrm{g} \mathrm{Zn} ; 14.79 \mu \mathrm{g} / \mathrm{g} \mathrm{Cr}$ and $4.40 \mu \mathrm{g} / \mathrm{g} \mathrm{Pb}$. Lead concentrations were comparable to those reported for moderately contaminated sites. The results showed that $\mathrm{Pb}$ is bioavailable to the bivalves, exhibiting high concentrations and exceeding both natural and reference values for human consumption. The freshwater bivalve Anodontites tenebricosus is a suitable biomonitor of contamination by metals.
\end{abstract}

Keywords: contamination; Ribeira de Iguape River; Anodontites tenebricosus.

\section{INTRODUÇÃO}

A região do Vale do Ribeira, entre o extremo nordeste do estado do Paraná e o sudeste do estado de São Paulo, foi palco de intensa atividade de mineração, tendo sido exploradas nove minas, cujo foco de interesse principal era a obtenção de $\mathrm{Pb}$ e, secundariamente, $\mathrm{Ag}$ e Au. As minas operaram de 1920 a 1995, sendo que a usina Plumbum - Mineração e Metalurgia S.A., localizada no município de Adrianópolis (PR), operou de 1945 a 1995. Nesse período, a Plumbum processou cerca de 3 milhões de toneladas de minério de $\mathrm{Pb} .{ }^{1}$

As condições de mineração e refino no Vale do Ribeira foram quase sempre rudimentares, não havendo controle sobre os impactos ambientais gerados durante as fases extrativa e de beneficiamento do minério. No período de 1945 a 1991, todos os resíduos sólidos da Plumbum foram lançados diretamente no Rio Ribeira de Iguape, sem tratamento, correspondendo a aproximadamente 5,5 t/ano (ou cerca de 253 toneladas, no total) de elementos tóxicos (As, Ba, Cd, $\mathrm{Pb}, \mathrm{Cu}, \mathrm{Cr}$ e $\mathrm{Zn}){ }^{1}$

Em estudos realizados durante o período das atividades de mineração e de beneficiamento do minério, décadas de 1980 e início de 1990, foram constatadas altas concentrações de $\mathrm{Pb}$ nas águas e sedimentos do Rio Ribeira de Iguape, em seus afluentes e no sistema estuarino lagunar Iguape-Cananéia. ${ }^{2-5}$ Como exemplo, tem-se o caso do Ribeirão do Rocha, que chegou a apresentar concentrações de $\mathrm{Pb}$ superiores a 730 vezes o limite máximo recomendado para a prevenção da vida aquática, além de concentrações de $2.560 \mathrm{mg} / \mathrm{kg}$ desse mesmo metal no sedimento de fundo. ${ }^{6}$

Análises isotópicas realizadas nos sedimentos coletados no Rio Ribeira de Iguape e no sistema estuarino lagunar Iguape-Cananéia

\footnotetext{
*e-mail: valguima@sc.usp.br
}

comprovaram que as fontes de contaminação nesse sistema aquático estavam associadas aos resíduos da mineração produzidos no Alto Vale do Ribeira pela Plumbum. . $7,8^{-1}$

Com o passar dos anos, as concentrações de $\mathrm{Pb}$ detectadas nos sedimentos diminuíram consideravelmente, como comprovado pelo mapeamento geoquímico de baixa densidade realizado desde as cabeceiras do rio até o município de Registro. ${ }^{9}$ A água do Rio Ribeira de Iguape também não é mais considerada fonte de risco para a população, pelo consumo direto, pois as concentrações dos metais estão abaixo dos limites legais. ${ }^{10}$

Mesmo tendo ocorrido diminuição das concentrações de metais nos sedimentos e na água do Rio Ribeira, recentemente, foi constatada a incorporação de elementos tóxicos pelos moluscos filtradores da espécie invasora Corbicula flumínea,${ }^{11}$ conhecida internacionalmente como monitor biológico da contaminação de ambientes fluviais. Os mesmos autores verificaram que a escória de fundição que foi lançada no Rio Ribeira de Iguape é cominuída ao longo desse sistema fluvial e é incorporada ao sedimento em suspensão, ${ }^{12,13}$ sendo assim assimilada pela espécie Corbicula flumínea. ${ }^{11}$

A incorporação de metais pela biota a partir dos sedimentos é frequente. No estuário do Rio Subaé amostras de peixes e moluscos ${ }^{14}$ apresentaram concentrações de $\mathrm{Pb}$ e $\mathrm{Cd}$ acima dos níveis aceitáveis para consumo humano. A principal fonte de contaminação desse estuário foi a Plumbum Mineração e Metalurgia S.A. (mesma empresa que contaminou o Rio Ribeira de Iguape), localizada em Santo Amaro da Purificação, no Recôncavo Baiano, às margens do Rio Subaé, que depositou nas proximidades desse rio escórias de fundição com elevadas concentrações de metais tóxicos. ${ }^{15}$

A biota tornou-se uma importante ferramenta em programas de monitoramento ambiental, uma vez que a biodisponibilidade dos contaminantes é medida diretamente. Moluscos bivalves têm 
sido extensivamente empregados na avaliação da contaminação de ambientes aquáticos ${ }^{16-20}$ por metais e outros contaminantes, pois fornecem informações robustas e integradas sobre o impacto ambiental e biodisponibilidade de tais elementos. Esses organismos são adequados como monitores biológicos de áreas contaminadas, pois eles são: sésseis, filtradores, de fácil coleta, estão presente ao longo de todo o ano e respondem rapidamente às variações das concentrações de metais biodisponíveis no meio. ${ }^{21}$

O bivalve límnico Anodontites tenebricosus ${ }^{22}$ (Lea, 1834) foi encontrado na Bacia do Rio Ribeira de Iguape, tanto nas áreas sem influência da mineração como nas áreas influenciadas por tais atividades. Entretanto, Anodontites tenebricosus é uma espécie nativa de bivalve cujo potencial bioindicador ainda não foi estudado. Essa espécie foi originalmente descrita por Bonetto, ${ }^{23}$ na Bacia do Rio da Prata, tendo sido registrada também nas Bacias dos Rios Uruguai, Paraná, Sapucaî ${ }^{24}$ e Rio dos Sinos. ${ }^{25}$

Assim sendo, este trabalho teve por objetivo avaliar se esta espécie de bivalve está incorporando os metais tóxicos $(\mathrm{Cd}, \mathrm{Pb}, \mathrm{Zn}$ e $\mathrm{Cr}$ ) associados aos resíduos de mineração (principalmente, com a escória) que foram lançados neste rio, ou seja, se tais metais estão biodisponíveis para essa espécie de bivalve e se a mesma é apropriada para ser empregada como monitor biológico.

\section{PARTE EXPERIMENTAL}

\section{Amostragem}

A investigação foi desenvolvida na Bacia Hidrográfica do Rio Ribeira de Iguape, em decorrência do histórico de contaminação deste rio por atividade de mineração. Para realização deste estudo, foram coletadas e analisadas amostras de Anodontites tenebricosus, espécie nativa de bivalve de água doce.

Exemplares de A. tenebricosus foram coletados em 5 pontos ao longo do rio (Figura 1S, material suplementar), sendo o CR localizado a montante da porção contaminada pela atividade de mineração (amostra de referência - background); IR e PT apresentando influência das atividades de mineração realizadas na mina do Rocha e da usina de beneficiamento do Rocha; IP e SI com influência de todas as atividades de mineração realizadas no Vale do Ribeira e da usina de beneficiamento Plumbum.

A amostragem foi realizada manualmente através do exame tátil do sedimento de fundo, durante o período climático de baixa pluviosidade (agosto e setembro de 2009), sendo coletados de 3 a 5 indivíduos por ponto de amostragem, em decorrência da dificuldade de obtenção dos mesmos, na época de coleta. Os exemplares coletados foram acondicionados em sacos plásticos fechados a vácuo e receberam a sigla ANO mais a terminologia do local de coleta. As amostras foram armazenadas em caixa de isopor com gelo, e no laboratório foram congeladas até o momento das análises.

Anodontites tenebricosus apresenta forma elíptica reniforme, altura média de 2,0 cm e comprimento médio de $4,0 \mathrm{~cm}$. Não foi possível separar os exemplares coletados em cada ponto de amostragem por diferença de tamanho, pois todos os indivíduos amostrados apresentaram tamanhos similares.

\section{Preparação das amostras}

As amostras foram inicialmente descongeladas à temperatura ambiente e pesadas. A parte mole (tecido) foi retirada com auxílio de uma espátula de politetrafluoretileno, lavada com água bidestilada, seca parcialmente em papel de filtro, pesada e congelada até a data da análise, conforme metodologia empregada em Tomazelli. ${ }^{26}$ Antes de serem analisadas, as amostras congeladas foram liofilizadas e pe- sadas, sendo a seguir maceradas em almofariz de ágata, assegurando a homogeneização do pó.

As amostras liofilizadas, para um mesmo ponto de amostragem, foram agrupadas ( $\mathrm{pool}$ ), visando obter quantidade de tecido suficiente para a digestão e análise.

\section{Digestão ácida das amostras}

Na preparação prévia das amostras para posterior determinação analítica utilizou-se o método da digestão ácida em sistema de micro-ondas. ${ }^{27}$ Primeiramente, pesaram-se aproximadamente cerca de $200 \mathrm{mg}$ de tecido liofilizado ao qual adicionaram-se, em frasco de politetrafluoretileno próprio para micro-ondas, $3 \mathrm{~mL}$ de ácido nítrico (Subboiling) e $2 \mathrm{~mL}$ de peróxido de hidrogênio (Suprapur ${ }^{\circledR}$ Merck), a seguir realizou-se a pré-digestão overnight em temperatura ambiente. Após a pré-digestão overnight, adicionaram-se $3 \mathrm{~mL}$ de água ultrapura (Milli- ${ }^{\circledR}$ ), o frasco de politetrafluoretileno foi selado e a amostra foi digerida em forno de micro-ondas (modelo Mars 5 da CEM Corporation). Os parâmetros empregados na digestão realizada em forno de micro-ondas foram: potência de $600 \mathrm{~W}, 100 \%$, tempo de rampa de $9 \mathrm{~min}$, temperatura de $145^{\circ} \mathrm{C}$ e tempo de permanência de 5 min a $145^{\circ} \mathrm{C}$.

Todo material utilizado foi descontaminado previamente às análises com água (Milli-Q ${ }^{\circledR}$ ) e ácido nítrico $10 \%$.

\section{Procedimento analítico}

A determinação quantitativa dos metais $(\mathrm{Cd}, \mathrm{Pb}, \mathrm{Cr}$ e $\mathrm{Zn})$ foi realizada em espectrômetro de massas de alta resolução com fonte de plasma indutivamente ativado (HR-ICPMS); element 1 marca Finnigan MAT (Bremen, Alemanha). Esse instrumento apresenta um sistema analisador de dupla focalização de íons, com geometria reversa (Nier-Johnson): um analisador magnético seguido de um analisador eletrostático. Esse tipo de equipamento pode operar em três modos nominais de resolução: baixa resolução $(\mathrm{m} / \Delta \mathrm{m}=300)$, média resolução $(\mathrm{m} / \Delta \mathrm{m}=3000)$ e alta resolução $(\mathrm{m} / \Delta \mathrm{m}=7500) . \mathrm{Pb}$ e Cd foram analisados no modo denominado baixa resolução $(\mathrm{m} / \Delta \mathrm{m}$ = 300) monitorando-se os nuclídeos ${ }^{208} \mathrm{~Pb} \mathrm{e}{ }^{111} \mathrm{Cd}$, utilizando-se uma solução de $10 \mathrm{ng} / \mathrm{g}$ de In como padrão interno monitorado por meio do isótopo ${ }^{115} \mathrm{In}$. Cr e $\mathrm{Zn}$ foram analisados no modo denominado média resolução $(\mathrm{m} / \Delta \mathrm{m}=3000)$ monitorando-se os nuclídeos ${ }^{52} \mathrm{Cr}$ e ${ }^{66} \mathrm{Zn}$, utilizando-se uma solução de $10 \mathrm{ng} / \mathrm{g}$ de In como padrão interno monitorado por meio do isótopo ${ }^{115} \mathrm{In}$.

A exatidão e repetitividade do método foram avaliadas através do material de referência certificado SRM 1566 a (Oyster tissue National Institute of Standard and Technology, NIST) em triplicata para cada batelada de amostras.

A cada batelada foram preparadas amostras em branco, visando verificar possíveis contaminações provenientes deste processo.

\section{RESULTADOS E DISCUSSÃO}

Nos tecidos secos de A. tenebricosus foram detectados $\mathrm{Cd}$, $\mathrm{Pb}, \mathrm{Cr}$ e $\mathrm{Zn}$, sendo que os valores médios determinados para esses elementos foram da ordem de: 1,00; 4,40;14,79 e 152,89 $\mu \mathrm{g} / \mathrm{g}$, respectivamente.

A validação do método empregado foi avaliada utilizando o material de referência certificado (SRM 1566 a), cuja faixa de recuperação para os elementos analisados foi de $92,77-106,70 \%$, exceto para o $\mathrm{Cr}$, que foi de $76,92 \%$, entretanto, o resultado encontrado está dentro da faixa englobada pelo valor da incerteza expandida do Cr no certificado do material de referência $( \pm 0,46 \mu \mathrm{g} / \mathrm{g})$, conforme observado na Tabela 1. 
Tabela 1. Concentrações de metais tóxicos detectados nos tecidos (base seca) de Anodontites tenebricosus. Unidade de medida ( $\mu \mathrm{g} / \mathrm{g}$ )

\begin{tabular}{|c|c|c|c|c|}
\hline Amostras & $\begin{array}{c}\mathrm{Cd} \\
\mu \mathrm{g} / \mathrm{g}\end{array}$ & $\begin{array}{c}\mathrm{Pb} \\
\mu \mathrm{g} / \mathrm{g}\end{array}$ & $\begin{array}{c}\mathrm{Cr} \\
\mu \mathrm{g} / \mathrm{g}\end{array}$ & $\begin{array}{c}\mathrm{Zn} \\
\mu \mathrm{g} / \mathrm{g}\end{array}$ \\
\hline CR-ANO (Amostra de Referência - Background) & $0,45 \pm 0,09$ & $1,29 \pm 0,23$ & $11,26 \pm 1,10$ & $90,09 \pm 4,87$ \\
\hline IR-ANO & $0,71 \pm 0,16$ & $3,68 \pm 0,60$ & $18,26 \pm 3,10$ & $136,20 \pm 14,00$ \\
\hline PT-ANO & $1,69 \pm 0,34$ & $2,90 \pm 0,42$ & $26,59 \pm 5,32$ & $211,67 \pm 42,33$ \\
\hline IP-ANO & $1,05 \pm 0,29$ & $3,80 \pm 0,57$ & $9,33 \pm 1,69$ & $163,54 \pm 17,43$ \\
\hline SI-ANO & $1,09 \pm 0,30$ & $10,32 \pm 2,06$ & $8,50 \pm 1,09$ & $162,96 \pm 23,42$ \\
\hline SRM 1566a - Encontrado & $3,86 \pm 0,56$ & $0,396 \pm 0,054$ & $1,10 \pm 0,03$ & $770 \pm 3,6$ \\
\hline SRM 1566a - Certificado & $4,15 \pm 0,38$ & $\mathbf{0 , 3 7 1} \pm \mathbf{0 , 0 1 4}$ & $1,43 \pm 0,46$ & $830 \pm 57$ \\
\hline \% de Recuperação & 93,01 & 106,70 & 76,92 & 92,77 \\
\hline
\end{tabular}

As maiores concentrações de $\mathrm{Pb}$ (Tabela 1) foram detectadas nos indivíduos coletados nos pontos de amostragem IP e SI, ambos localizados no município de Iporanga, porção do rio a jusante de todas as atividades de mineração e das usinas de beneficiamento do minério. Esta mesma tendência, maiores concentrações de $\mathrm{Pb}$ nas amostras coletadas no ponto de amostragem localizado em Iporanga, foi descrita para a espécie Corbicula flumíne ${ }^{11}$ e para os sedimentos aluvionares. ${ }^{13}$

Para os outros elementos investigados ( $\mathrm{Cd}, \mathrm{Cr}$ e $\mathrm{Zn})$, as concentrações mais elevadas foram detectadas na amostra PT, com influência apenas da mina do Rocha e da usina de beneficiamento do Rocha (Tabela 1).

Não foi possível correlacionar diferenças de concentrações de metais com peso corporal, pois todos os indivíduos analisados apresentaram pesos similares. Em estudos realizados com Anodontites trapesialis não foram encontradas correlações significativas entre peso corporal e concentração de metais. ${ }^{28}$

Com relação às diferenças de concentrações de metais, outra correlação que não foi possível realizar com essa espécie foi com relação ao sexo, uma vez que pelas informações bibliográficas disponíveis (já que não se têm muitos dados sobre essa espécie) se acredita que a mesma é hermafrodita. A mesma tendência foi observada na Anodontites trapesialis. ${ }^{28}$

De maneira geral, as concentrações de $\mathrm{Pb}$ determinadas para a espécie Anodontites tenebricosus ultrapassaram os limites máximos de tolerância para contaminantes inorgânicos em peixes e produtos de pesca estabelecidos pela legislação brasileira para consumo humano (Portaria 658/98 da ANVISA, ${ }^{29}$ equivalente a 2,0 $\mu \mathrm{g} / \mathrm{g}$ para este metal), para todos os pontos de amostragem com exceção do ponto correspondente ao valor de background (CR-ANO), conforme observado na Figura 1.

Os bivalves coletados no ponto SI foram considerados os mais

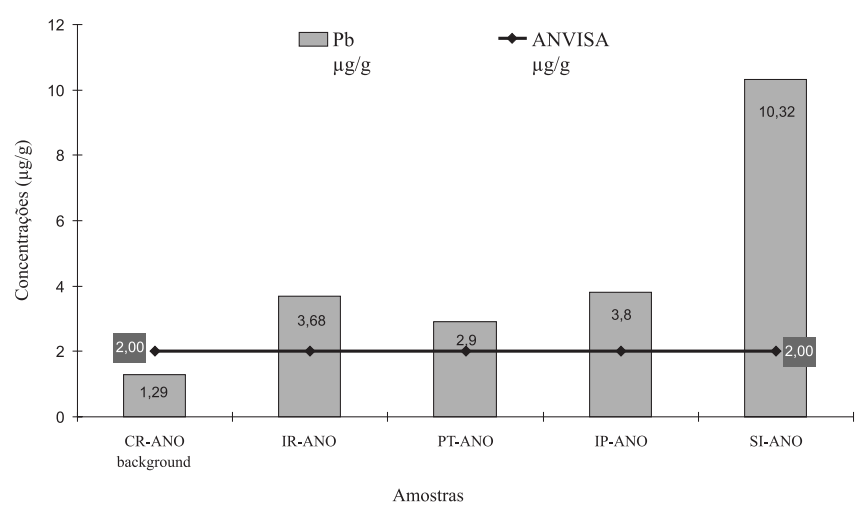

Figura 1. Comparação de Pb detectado na Anodontites tenebricosus com valor de referência da ANVISA enriquecidos por $\mathrm{Pb}$, ultrapassando em 5,16 vezes o valor definido pela portaria da ANVISA, comprovando que a porção do rio localizada em Iporanga (pontos IP e SI) é, ainda hoje, a mais enriquecida por $\mathrm{Pb}$ e que necessita de monitoramento constante.

As concentrações de $\mathrm{Pb}$ também foram comparadas com os valores de background ou branco da amostra (valor de referência obtido na área de estudo, a montante das atividades de mineração e beneficiamento, área sem influência da contaminação por atividades de mineração), sendo que todos os pontos de amostragem exibiram valores mais elevados que o background (Figura 2).

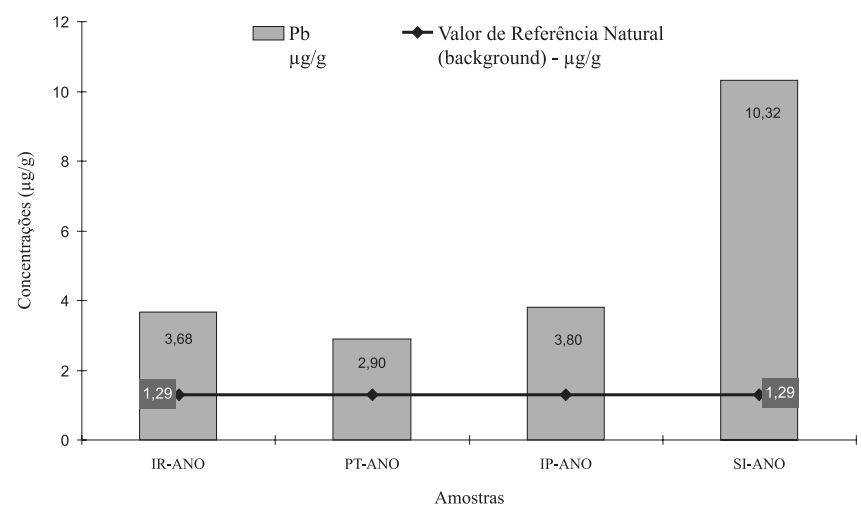

Figura 2. Comparação de Pb detectado na Anodontites tenebricosus com valor de referência natural (background)

$\mathrm{O} \mathrm{Pb}$ é um elemento tóxico não essencial que se acumula no organismo. Como esse metal afeta todos os órgãos e sistemas do organismo, os mecanismos de toxicidade propostos envolvem processos bioquímicos fundamentais, que incluem a habilidade do $\mathrm{Pb}$ de inibir ou imitar a ação do $\mathrm{Ca}$ e de interagir com proteínas. ${ }^{26}$ Estudos na região do Vale do Ribeira ${ }^{30}$ revelaram que grande parte da população local, residente na Vila Mota (vila de ex-trabalhadores da usina Plumbum), está contaminada por tal metal.

As concentrações mais elevadas de Cd para esta espécie de bivalve foram encontradas na amostra coletada no ponto PT (influência só das atividades de mineração e beneficiamento na mina do Rocha), correspondente a $1,69 \mu \mathrm{g} / \mathrm{g}$, estando acima do valor de referência definido pela Portaria 658/98 da ANVISA $(1,00 \mu \mathrm{g} / \mathrm{g}) .{ }^{29} \mathrm{Para}$ os pontos de amostragem IP e SI, as concentrações de Cd foram similares ao valor de referência definido pela Portaria 658/98 da ANVISA (Tabela 1).

A concentração de $\mathrm{Cd}$, para todos os pontos de amostragem, quando comparada com o valor de background $(0,45 \mu \mathrm{g} / \mathrm{g})$, também se encontra acima do mesmo. $\mathrm{O} \mathrm{Cd}$, assim como o $\mathrm{Pb}$, ocupa lugar de destaque entre os vários metais tóxicos associados à contaminação do ambiente aquático e que podem causar problemas de intoxicação ao homem e a outros organismos. Este metal é capaz de reagir com 
moléculas ligantes presentes nas membranas celulares, o que, muitas vezes, acaba conferindo propriedade de bioacumulação na cadeia alimentar, persistência no ambiente e distúrbios nos processos metabólicos dos seres vivos. ${ }^{26}$

As concentrações de $\mathrm{Zn}$ detectadas nos tecidos de A. tenebricosus encontram-se bem acima dos valores determinados na amostra de background natural $(90,09 \mu \mathrm{g} / \mathrm{g})$ (Tabela 1). Tal fato, provavelmente, pode estar associado à grande quantidade de escórias que foram lançadas neste rio, as quais continham quantidades elevadas de $\mathrm{Zn}^{13}$ que, possivelmente, foram retidas durante o ciclo alimentar dos bivalves. Durante o processo metalúrgico para obtenção do $\mathrm{Pb}$ refinado, foi adicionado $\mathrm{Zn}$ metálico fundido para separar $\mathrm{Ag}$, $\mathrm{Au}$ e $\mathrm{Cu}$ do $\mathrm{Pb}$ explicando, assim, a alta concentração de $\mathrm{Zn}$ na escória de fundição. A concentração média de Zn na escória é de 118.004,33 $\mathrm{mg} / \mathrm{kg}$, de acordo com Guimarães. ${ }^{13}$

Outra explicação para a elevada concentração de $\mathrm{Zn}$ pode estar ligada com as necessidades metabólicas dos bivalves para com esse elemento, uma vez que, nestes organismos, esse metal é requerido em grandes concentrações para o seu metabolismo, potencializando, assim, os processos de quelação com subsequente incorporação. ${ }^{31} \mathrm{O}$ valor médio de $\mathrm{Zn}(152,89 \mu \mathrm{g} / \mathrm{g})$ encontrado em A. tenebricosus está acima do estabelecido pelo Decreto $\mathrm{n}^{\circ} 55.871 / 65-\mathrm{GP}, 50 \mu \mathrm{g} / \mathrm{g}$ para o $\mathrm{Zn}$ em alimentos destinados ao consumo humano.
$\mathrm{O}$ teor de $\mathrm{Cr}$ variou de um ponto de coleta para outro, estando abaixo do valor de background natural nas amostras IP e SI (Tabela 1). A maior concentração de Cr foi observada na amostra PT. Segundo Guimarães, ${ }^{13}$ o Cr presente nos sedimentos do Rio Ribeira de Iguape é de origem geogênica. No mapeamento geoquímico realizado por Lopes Jr. ${ }^{9}$ nota-se que a concentração deste metal é elevada em toda a bacia hidrográfica, tanto nos sedimentos ativos de corrente como nos aluvionares. A concentração desse metal também é bastante elevada nas rochas da região. Mesmo sendo de origem natural, a partir das análises realizadas observa-se que este elemento está biodisponível e sendo incorporado pela biota, estando acima dos valores de referência natural $(11,26 \mu \mathrm{g} / \mathrm{g})$ para a espécie A. tenebricosus nos pontos de amostragem IR $(18,26 \mu \mathrm{g} / \mathrm{g})$ e PT $(26,59 \mu \mathrm{g} / \mathrm{g})$.

Além das comparações das concentrações com os valores de referência da ANVISA e com o valor de background natural, estes resultados também foram comparados com valores obtidos em outras áreas do mundo, onde estudos semelhantes foram realizados com moluscos bivalves de água doce de diferentes espécies (Tabela 2).

As concentrações de $\mathrm{Pb}$ detectadas na $A$. tenebricosus estão acima dos valores reportados para esse mesmo metal em áreas consideradas não contaminadas, para diferentes espécies de bivalves de água doce (Tabela 2). ${ }^{17,32}$ Entretanto, tais concentrações não atingiram níveis

Tabela 2. Comparação das concentrações de metais em tecidos de moluscos bivalves de água doce de diversas localidades, coletados tanto em áreas contaminadas como em não contaminadas. Intervalo de Concentração e Média \pm Desvio Padrão ( $\mu \mathrm{g} / \mathrm{g}$ em peso seco)

\begin{tabular}{|c|c|c|c|c|c|}
\hline Espécie/Local & $\begin{array}{c}\mathrm{Cd} \\
\mu \mathrm{g} / \mathrm{g}\end{array}$ & $\begin{array}{c}\mathrm{Pb} \\
\mu \mathrm{g} / \mathrm{g}\end{array}$ & $\begin{array}{c}\mathrm{Cr} \\
\mu \mathrm{g} / \mathrm{g}\end{array}$ & $\begin{array}{c}\mathrm{Zn} \\
\mu \mathrm{g} / \mathrm{g}\end{array}$ & Ref. \\
\hline $\begin{array}{l}\text { Elliptio complanata } \\
\text { Rio St. Lawrence (Canadá) }\end{array}$ & - & $1,25-2,2$ & - & $313-588$ & 32 \\
\hline $\begin{array}{l}\text { Anodonta anatina } \\
\text { Rio Tâmisa (Inglaterra) }\end{array}$ & $0,4-5,9$ & $9,8-42,5$ & - & $403,2-1870$ & 33 \\
\hline $\begin{array}{l}\text { Lampsilis ventricosa } \\
\text { Big River (USA) }\end{array}$ & $0,35-11,3$ & $0,46-74,2$ & - & - & 35 \\
\hline $\begin{array}{l}\text { Corbicula flumínea } \\
\text { Rio Shatt al-Arab (Iraque) }\end{array}$ & $11,6-53,1$ & $0,32-3,2$ & - & $32-79$ & 17 \\
\hline $\begin{array}{l}\text { Lamellidens marginalis } \\
\text { Rio Cauvery (Índia) }\end{array}$ & - & $6,4-12$ & - & $250-544$ & 36 \\
\hline $\begin{array}{l}\text { Dreissena polymorfa } \\
\text { Lago Maarseveen (Holanda) }\end{array}$ & $0,01-17$ & $0,3-1,5$ & - & $74-113$ & 37 \\
\hline $\begin{array}{l}\text { Dreissena polymorfa } \\
\text { Rio Rhine e Meuse (Europa Ocidental) }\end{array}$ & $0,1-6,5$ & $0,1-13$ & - & $80-170$ & 38 \\
\hline $\begin{array}{l}\text { Dreissena polymorfa } \\
\text { Rio de New York city (EUA) }\end{array}$ & $0,55-5,89$ & $1,03-4,3$ & $1,55-5,0$ & $94,4-276$ & 34 \\
\hline $\begin{array}{l}\text { Lampsilis radiata } \\
\text { Rio St. Lawrence (Canadá) }\end{array}$ & 0,51 & 6,07 & 12,2 & 448 & 39 \\
\hline $\begin{array}{l}\text { Corbicula flumínea } \\
\text { Rio de La Plata (Argentina) }\end{array}$ & $1,1 \pm 0,4$ & - & $5,2 \pm 2,8$ & $197 \pm 54$ & 18 \\
\hline $\begin{array}{l}\text { Anodontites trapesialis } \\
\text { Bacia do Rio Piracicaba (Brasil) }\end{array}$ & $0,77-2,83$ & $10,78-20,7$ & $4,23-12,5$ & $304-579$ & 40 \\
\hline $\begin{array}{l}\text { Anodontites trapesialis } \\
\text { Rio Pardo (Brasil) }\end{array}$ & $0,1-2,95$ & $0,8-29$ & $0,2-18,6$ & $66-722$ & 26 \\
\hline $\begin{array}{l}\text { Corbicula flumínea } \\
\text { Rio Ribeira de Iguape (Brasil) } \\
\text { PLUMBUM de Adrianópolis }\end{array}$ & 0,71 & 2,41 & 7,11 & 177,21 & 11 \\
\hline $\begin{array}{l}\text { Anodontites tenebricosus } \\
\text { Rio Ribeira de Iguape (Brasil) } \\
\text { PLUMBUM de Adrianópolis }\end{array}$ & 1,0 & 4,40 & 14,79 & 152,89 & Este estudo \\
\hline $\begin{array}{l}\text { Sururu } \\
\text { Rio Subaé (Brasil) } \\
\text { PLUMBUM de Santo Amaro }\end{array}$ & 1,06 & 1,15 & - & - & 14 \\
\hline
\end{tabular}


tão elevados como os reportados em áreas contaminadas, conforme observado na Tabela $2 .^{33}$

A concentração de $\mathrm{Cd}$ determinada no tecido de A. tenebricosus está dentro dos limites detectados em bivalves coletados em áreas não contaminadas, ou como limite inferior da concentração determinada em região contaminada (Tabela 2 ). ${ }^{18}$

As concentrações de $\mathrm{Zn}$ detectadas na A. tenebricosus estão acima dos valores reportados para esse metal em moluscos coletados em áreas consideradas não contaminadas (Tabela 2). ${ }^{17}$ Por outro lado, essas concentrações não atingiram níveis tão elevados como os determinados em áreas contaminadas. ${ }^{32}$

Já a concentração de $\mathrm{Cr}$ obtida para A. tenebricosus está acima dos valores definidos para outras espécies de bivalves, tanto no Brasil como em outros países, ${ }^{18,34}$ tanto para áreas contaminadas como para não contaminadas.

$\mathrm{Na}$ Tabela 2, nota-se ainda que as concentrações de $\mathrm{Cd}, \mathrm{Pb}$ e $\mathrm{Cr}$ foram mais elevadas para a espécie Anodontites tenebricosus que para a Corbicula flumínea, ${ }^{11}$ ambas coletadas no Rio Ribeira de Iguape. Nesse mesmo rio, apenas a concentração de $\mathrm{Zn}$ foi mais elevada para a $C$. fluminea que para a $A$. tenebricosus.

O Rio Subaé passou por processo de contaminação semelhante ao ocorrido no Rio Ribeira de Iguape, onde escórias de fundição enriquecidas, principalmente, em $\mathrm{Pb}, \mathrm{Zn}$ e $\mathrm{Cd}$ foram lançadas ou depositadas nas proximidades desse rio. Em consequência, nos dois rios, nota-se a biodisponibilidade de $\mathrm{Pb}$ e $\mathrm{Cd}$ e a incorporação dos mesmos pela biota (Tabela 2), mesmo tendo ocorrido diminuição desses metais nos sedimentos e na água.

Esta comparação entre as concentrações de metais em tecidos de diferentes espécies de bivalves de água doce foi realizada visando obter dados em ambientes contaminados e não contaminados. No entanto, há certa restrição quanto a essa comparação, em face da mesma ser empregada genericamente e não especificamente para a mesma espécie, existindo variabilidade das condições fisiológicas dos organismos e dos parâmetros físico-químicos do ambiente.

\section{CONCLUSÕES}

Com os resultados obtidos pôde-se concluir que os metais potencialmente tóxicos lançados na forma de resíduos da mineração no Rio Ribeira de Iguape continuam biodisponíveis e estão sendo incorporados pela espécie de bivalve nativo A. tenebricosus.

A espécie Anodontites tenebricosus foi considerada apropriada para ser empregada como monitor biológico da contaminação de sistemas fluviais, pois apresenta as características necessárias para um biomonitor: ser séssil, fornecer tecido suficiente para a realização das análises, ser filtrador e resistente ao acúmulo de metais.

Com relação ao Rio Ribeira de Iguape, as concentrações de $\mathrm{Pb}$, $\mathrm{Cd}$ e $\mathrm{Cr}$ foram mais elevadas para a espécie Anodontites tenebricosus que para a Corbicula flumínea, anteriormente analisada, confirmando o fato que a A. tenebricosus é um bom monitor biológico.

Dos metais tóxicos investigados, o $\mathrm{Pb}$ é o que exibiu valores mais preocupantes, estando acima dos valores da ANVISA. Esse metal também exibiu concentrações comparáveis com as reportadas em estudos realizados em áreas moderadamente contaminadas. $\mathrm{O} \mathrm{Pb}$ é um metal não essencial, sendo que a exposição a esse metal produz diversos efeitos nocivos aos organismos aquáticos. A porção próxima ao município de Iporanga foi a que exibiu maiores concentrações $\mathrm{de} \mathrm{Pb}$ nos tecidos de Anodontites tenebricosus; isto ocorreu em decorrência deste ponto estar sob influência direta de todas as minas e usinas de beneficiamento do Vale do Ribeira. Mesma tendência já havia sido reportada por Guimarães, para a espécie Corbicula flumínea, neste mesmo rio.

Foram observadas altas concentrações de $\mathrm{Cd}, \mathrm{Zn}$ e $\mathrm{Cr}$ no ponto próximo à mina do Rocha, demonstrando a persistência de metais tóxicos nesta área, mesmo após a desativação da mina e retirada da pilha de rejeito do beneficiamento da área pertencente à usina de beneficiamento do Rocha. A concentração de Cd detectada neste ponto está acima do valor de referência definido pela Portaria 658/98 da ANVISA $(1,00 \mu \mathrm{g} / \mathrm{g})$ e as concentrações de $\mathrm{Zn}$ e Cr estão muito acima das encontradas na amostra de background natural, necessitando de monitoramento constante.

De fato, a concentração de $\mathrm{Cr}$ no Rio Ribeira de Iguape está elevada, como comprovado pela comparação dessas concentrações com valores detectados em bivalves coletados em diferentes áreas, tanto no Brasil como no mundo, indicando que mesmo esse elemento sendo de origem natural a biota o está incorporando.

De maneira geral, pôde-se concluir que os metais ainda hoje estão biodisponíveis e que os organismos viventes no Rio Ribeira de Iguape estão incorporando tais metais, principalmente o $\mathrm{Pb}$. Tal fato indica a necessidade de monitoramento e estudos detalhados em outros organismos viventes no Vale do Ribeira, pois a A. tenebricosus é consumida pela população ribeirinha e como qualquer outro organismo, faz parte da teia trófica, e sendo bivalve bentônico, deve fazer parte da dieta de peixes e aves, portanto, os metais podem estar sofrendo migração e transferência na cadeia trófica.

\section{MATERIAL SUPLEMENTAR}

Está disponível em http://quimicanova.sbq.org.br, na forma de arquivo PDF, com acesso livre. A Figura 1S apresenta o mapa de localização da área de estudo, juntamente com os pontos de amostragem dos exemplares de Anodontites tenebricosus no Rio Ribeira de Iguape.

\section{AGRADECIMENTOS}

À Fundação de Amparo a Pesquisa do Estado de São Paulo (FAPESP) pela concessão da bolsa de estudo de pós-doutoramento (Processo 08/54607-5) e auxílio individual à pesquisa (Processo 09/52762-6).

\section{REFERÊNCIAS}

1. Cassiano, A. M.; Tese de Doutorado, Universidade de São Paulo, Brasil, 2001.

2. Eysink, G. G. J.; Pádua, H. B.; Piva-Bertoletti, S. A. E.; Martins, M. C.; Pereira, D. N.; Rev. Cetesb 1988, 2, 13

3. Corsi, A. C.; Landim, P. M. B.; Rev. Geoc. UNESP 2003, 22, 61.

4. Tessler, M. G.; Suguio, K.; Robiolotta, P. R.; An. Acad. Bras. Cienc. 1987, 2, 263.

5. Moraes, R. P.; Dissertação de Mestrado, Universidade Estadual de Campinas, Brasil, 1997.

6. Companhia de Tecnologia de Saneamento Ambiental - Cetesb; Relatório Final, São Paulo, 1986.

7. Moraes, R. P.; Figueiredo, B. R.; Lafon, J. M.; Terrae 2004, 1, 26.

8. Magalhães, N.; Babinski, M.; Guimarães, V.; Sígolo, J. B.; Resumos do $45^{\circ}$ Congresso Brasileiro de Geologia, Belém, Brasil, 2010.

9. Lopes, Jr., I.; Atlas Geoquímico do Vale do Ribeira, CPRM: São Paulo, 2005.

10. http://www.who.int/water_sanitation_health/dwq/fulltext.pdf, acessada em Março 2011.

11. Guimarães, V.; Sígolo, J. B.; Quim. Nova 2008, 31, 1696.

12. Guimarães, V.; Sígolo, J. B.; Geol. USP 2008, 8, 10.

13. Guimarães, V.; Tese de Doutorado, Universidade de São Paulo, Brasil, 2007

14. Cunha, P. S. P.; Araújo, P. S. P.; Laudo Pericial, Bahia, 2001. 
15. Machado, S. L.; Ribeiro, L. D.; Kipertok, A.; Botelho, M. A. B.; Carvalho, M. F.; Eng. Sanit. Ambient. 2004, 2, 155.

16. Tessier, A.; Campbell, P. G. C.; Can. J. Fish. Aquat. Sci. 1984, 41, 1463.

17. Abaychi, J. K.; Mustafá, Y. Z.; Environ. Pollut. 1988, 54, 109.

18. Bilos, C.; Colombo, J. C.; Presa, M. J. R.; Environ. Pollut. 1998, 99, 1.

19. Foster, R. B.; Bates, J. M.; Environ. Sci. Technol. 1978, 12, 962.

20. Phillips, D. J. H.; Rainbow, P. S.; Biomonitoring of trace aquatic contaminants, Chapman \& Hall: London, 1994.

21. Rezende, C. E.; Lacerda, L. D.; Rev. Brasil. Biol. 1986, 46, 247.

22. Lea, I.; Am. Phil. Soc. Trans. 1834, 5, 119.

23. Bonetto, A. A.; Physis 1967, 26, 467.

24. Viana, M. P.; Avelar, W. E. P.; Resumos do IX Congresso de Ecologia do Brasil, São Lourenço, Brasil, 2009.

25. Mansur, M. C. D.; Pereira, D.; Rev. Bras. Zool. 2006, 23, 1147.

26. Tomazelli, A. C.; Tese de Doutorado, Universidade de São Paulo, Brasil, 2003.

27. Ashoka, S.; Peake, B. M.; Bremner, G.; Hageman, K. J.; Reid, M. R.; Anal. Chim. Acta 2009, 653, 191.

28. Tomazelli, A. C.; Martinelli, L. A.; Avelar, W. E. P.; Camargo, P. B.; Fostier, A.; Ferraz, E. S. B.; Krug, F. J.; Júnior, D. S.; Braz. Arch. Biol. Technol. 2003, 46, 684.
29. http://www.anvisa.gov.br/legis/index.htm, acessada em Fevereiro 2011.

30. http://www.comciencia.br/reportagens/2005/11/09_impr.shtml, acessada em Fevereiro 2011

31. Rojas, M. O. A. I.; Cavalcante, P. R. S.; Souza, R. C.; Dourado, E. C. S.; Bol. Lab. Hidrobiol. 2007, $20,8$.

32. Manly, R.; George, W. O.; Environ. Pollut. 1977, 14, 154.

33. Dermott, R. M.; Lum, K. R.; Environ. Pollut. 1986, 12, 143.

34. Secor, C. L.; Mills, E. L.; Harshbarger, J.; Kuntz, H. T.; Gutenmann, W. H.; Lisk, D. J.; Chemosphere 1993, 26, 1575.

35. Czarnezki, J. M.; Bull. Environ. Contam. Toxicol. 1987, 38, 646.

36. Hameed, P. S.; Raj, A. I. M.; Chem. Ecol. 1990, 4, 64.

37. van Hattun, B.; Timmermans, K. R.; Govers, H. A.; Environ. Toxicol. Chem. 1991, 10, 292.

38. Kraak, M. H. S.; Scholten, M. C. T. H.; Peeters, W. H. M.; Kock, W. C.; Environ. Pollut. 1991, 74, 114.

39. Metcalfe-Smith, J. L.; Green, R. H.; Grapentine, L. C.; Can. J. Fish. Aquat. Sci. 1996, 53, 219.

40. Tomazelli, A. C.; Dissertação de Mestrado, Universidade de São Paulo, Brasil, 1999. 
USO DO BIVALVE LÍMNICO Anodontites tenebricosus (LEA, 1834) NO BIOMONITORAMENTO DE METAIS DO RIO RIBEIRA DE IGUAPE

Valéria Guimarães Silvestre Rodrigues*

Departamento de Geotecnia, Escola de Engenharia de São Carlos, Universidade de São Paulo, Av. Trabalhador São-carlense, 400 , 13566-590 São Carlos - SP, Brasil

Aline Fujikawa e Denis Moledo de Souza Abessa

Universidade Estadual Paulista "Júlio de Mesquita Filho", Campus Experimental do Litoral Paulista, Pça. Infante D. Henrique, s/no, 11330-900 São Vicente - SP, Brasil

Marcos Antonio Hortellani e Jorge Eduardo Souza Sarkis

Instituto de Pesquisas Energéticas e Nucleares, Av. Lineu Prestes, 2242, 05508-000 São Paulo - SP, Brasil

Joel Barbujiani Sígolo

Departamento de Geologia Sedimentar e Ambiental, Instituto de Geociências, Universidade de São Paulo, Rua do Lago, 562, 04280-000 São Paulo - SP, Brasil

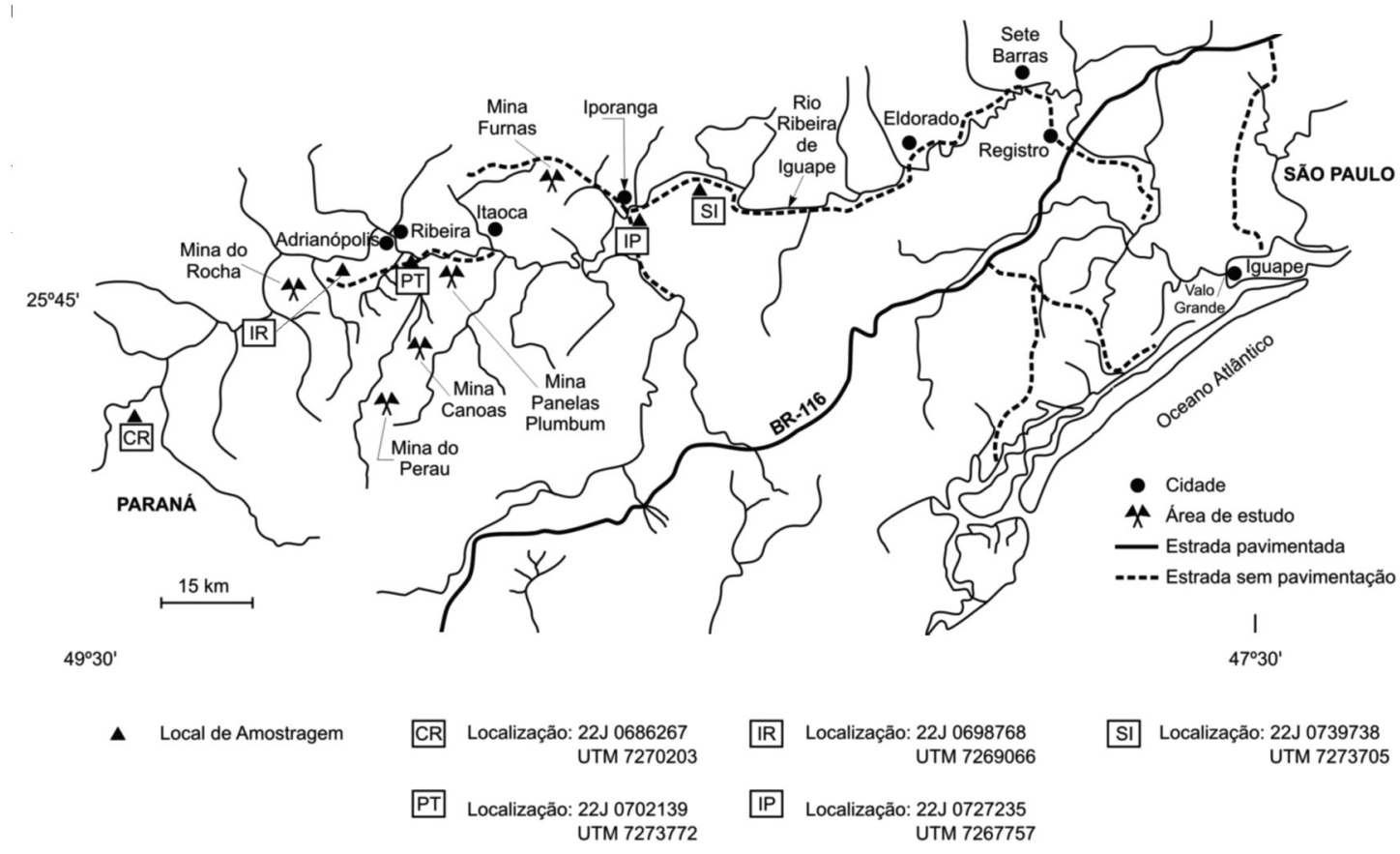

Figura 1S. Mapa de localização dos pontos de amostragem de Anodontites tenebricosus - Rio Ribeira de Iguape. Modificado da ref. 5

*e-mail: valguima@sc.usp.br 